\title{
A computer-controlled olfactometer for fMRI and electrophysiological studies of olfaction
}

\author{
TYLER S. LORIG and DAVID G. ELMES \\ Washington and Lee University, Lexington, Virginia \\ and \\ DAVID H. ZALD and JOSE V. PARDO \\ University of Minnesota, Minneapolis, Minnesota \\ and Minneapolis Veterans Administration Hospital, Minneapolis, Minnesota
}

\begin{abstract}
A design for an inexpensive and reliable olfactometer is presented. The design has several advantages for fMRI and electrophysiology investigators. These advantages include relatively rapid odorant rise times, computer control, multiple odor administration, and no ferrous materials near the subjects. In addition, the device is contamination resistant, and, because the air is neither warmed nor humidified, it is unlikely to become an incubator for bacteria. The olfactometer is constructed of off-the-shelf chromatography parts that require little modification.
\end{abstract}

A variety of instruments have been developed over the past 100 years for the accurate and metered presentation of odors (for a review, see Prah, Sears, \& Walker, 1995). Most of these instruments have been developed to meet the demands of particular situations or unique experimental settings, with each new need generating changes to existing devices. For instance, Kobal and Hummel (1988) developed an olfactometer with very rapid rise times in order to produce chemosensory event-related potentials. Laing (1986) also produced a unique olfactometer, for behavioral work with odor mixtures, that allowed odors to be introduced in very rapid succession. The advent of fMRI studies of olfactory and chemosensory processes presents a new set of needs, and the olfactometer described here has been developed to meet these needs in a simple and cost-effective manner.

The fMRI laboratory presents several important and new demands on an olfactometer. The most obvious and essential is the absence of ferrous metal near the magnet. Ideally, the instrument should have the following features: (1) computer control; (2) effective delivery of a variety of odors, in series or randomly; (3) production of an odor stimulus of selectable and reliable duration in a constant airstream, without any additional type of ancillary stimulation (e.g., tactile, auditory); (4) resistance to contamination; (5) durability; (6) ease of operation, refilling, and cleaning; and (7) low cost. The olfactometer described here meets these needs and is relatively easy to construct. With the exception of excluding ferrous metals,

The authors thank M. L. Tarpley for his assistance in obtaining the glass balls and A. Livermore for his comments on a draft of this manuscript. We also thank K. Theus, L. Salerno, and S. McGeorge, who were instrumental in the collection of the CSERP data. Correspondence concerning this should be addressed to T. S. Lorig, Department of Psychology, Washington and Lee University, Lexington, VA 24450-0303 (e-mail: tlorig@,wlu.edu). these needs are also common to laboratories studying chemosensory event-related potentials (CSERPs) and, in some cases, laboratories that study behavioral responses to odors. Thus, this olfactometer may have applications in a variety of experimental settings.

\section{OPERATION}

The olfactometer is based on a series of computercontrolled Teflon solenoid valves with switching times of $15 \mathrm{msec}$ (Cole-Parmer, Chicago, IL). The arrangement of the valves and flowmeters can be seen in Figure 1.

Air from a compressor used for home respiratory care (Bunn BA/400A) provides the air supply for the olfactometer's operation. This air is filtered to remove odors and particulates and then enters a series of flowmeters. The constant flow channel goes directly to the subject and provides a carrier airstream that adds to the odorant stream. In addition, the flow through this channel continuously washes the common odor-bearing portions of the olfactometer with clean air.

In the nonactive state, the constant flow is combined with the flow through the control channel. During odor administration, the control channel is vented outside the olfactometer, and the flow through one of the odor channels is briefly directed to the subject. After the stimulation interval has ended, the control flow is redirected to the subject. These two flows (constant + control or constant + odor) make up the total amount of air that reaches the subject. If, for instance, one wished to administer a total of $3 \mathrm{~L} / \mathrm{min}$ of air, $1 \mathrm{~L} / \mathrm{min}$ might be used in the constant flow channel and $2 \mathrm{~L} / \mathrm{min}$ in the control channel. This would mean that the total flow in each of the odor channels should also be set to $2 \mathrm{~L} / \mathrm{min}$.

Each odor channel (identified in Figure 1 by Roman numerals) consists of two flowmeters and an odor vessel. 


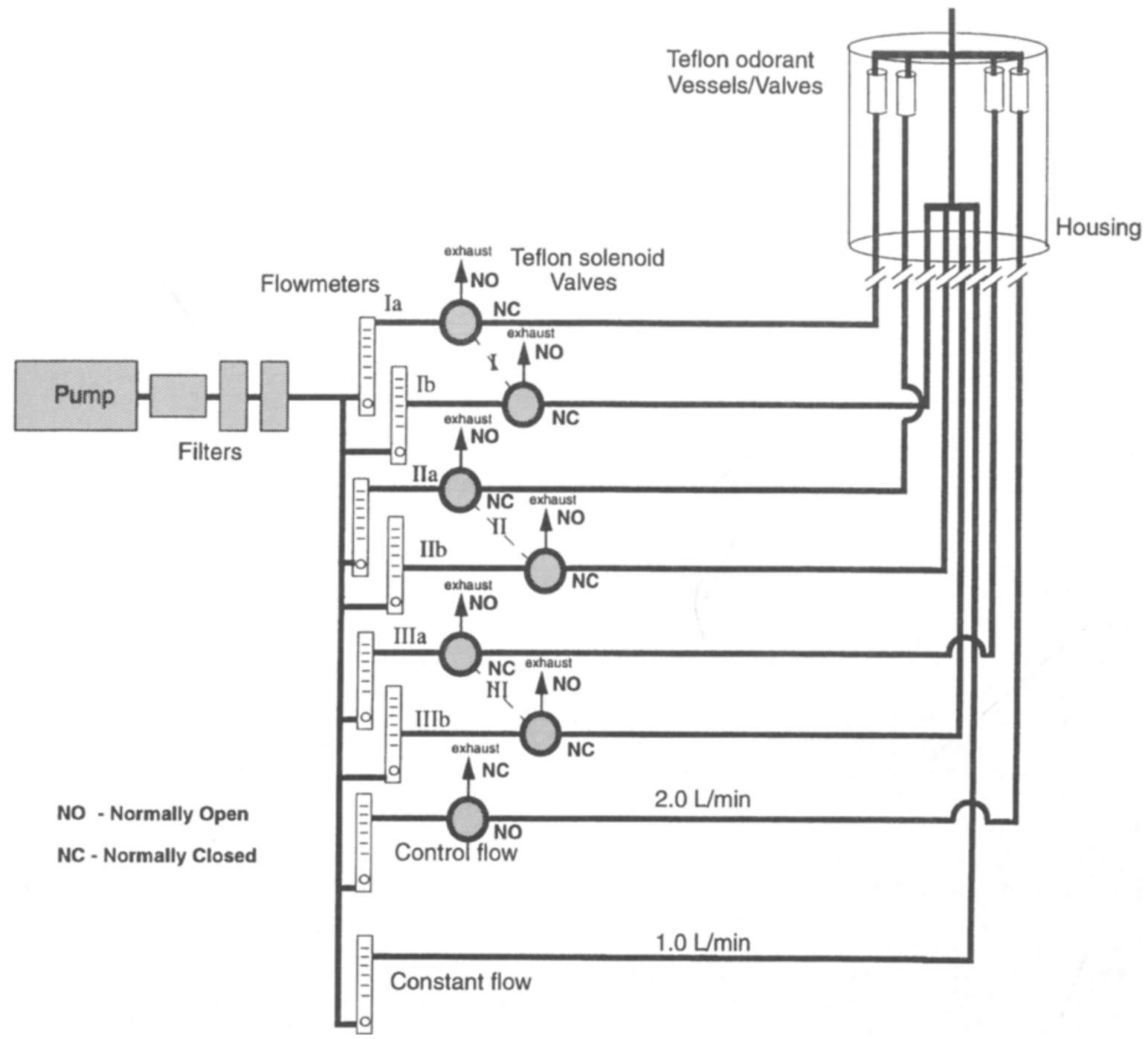

Figure 1. Schematic diagram of the olfactometer. Roman numerals refer to the individual channels. Note that the valve for the control flow is normally open.

Only one of the flowmeters is directly connected to the odor vessel. That meter controls the amount of air that actually passes over the odorant. The other flowmeter is used to dilute the airstream. If one wished to reduce by half the concentration of the odor presented to the subject and wished to maintain a total air flow of $2 \mathrm{~L} / \mathrm{min}$ in the odor channel, each flowmeter would be set to deliver $1 \mathrm{~L} / \mathrm{min}$. If a $75 \%$ odor dilution was sought, the flowmeter connected to the odor would be set to $1.5 \mathrm{~L} / \mathrm{min}$, and the diluting channel set to $0.5 \mathrm{~L} / \mathrm{min}$. If $100 \%$ of the air was to be directed through the odorant, that flowmeter would be set to $2 \mathrm{~L} / \mathrm{min}$, and the diluting channel to $0 \mathrm{~L} / \mathrm{min}$. The total flow in these two odor channels should always match that in the control channel. If this matching is not maintained, differences in flow will result, and the subject may perceive a tactile cue from the flow change.

The odor vessels in this design are constructed of small $(16 \times 50 \mathrm{~mm})$ Teflon cylinders with fittings at each end.
These vessels have been modified to contain two glass ball valves on either side of the odor. The odorant, in solution, is placed on filter paper inside the cylinder. When the computer initiates a stimulation, a solenoid valve opens that passes the air from the flowmeter connected to the cylinder over the odorant and into a Teflon manifold connected to a nosepiece. The positive pressure provided by the solenoid opening causes the ball valves in the odor cylinder to open. The air from the constant channel and the diluent channel also enters the manifold and mixes with the odorized air to dilute the sample before entering the subject's nose. The ball valves in other odor cylinders block the odorized and diluent air from entering any of the other cylinders and force it to be evacuated through the nosepiece. Because the cylinders have no ferrous parts, they can be placed close to the subject when inside the fMRI magnet. This means that the distance from the odorant to the subject is short, and lit- 
tle adhesion or contamination can occur in the olfactometer. It also means that the onset of the odor will be faster and more square-wave-like because of decreased diffusion of the odor bolus into the surrounding air inside the tubing. Because the odors are contained near the subject, the tubing supplying the cylinders can be long, and thus, the ferrous elements-such as the solenoids, flowmeters, pump, and chassis of the olfactometer-can be some distance from the subject and magnet. In our device, the tubing is $7 \mathrm{~m}$ long.

\section{CONSTRUCTION}

With only one exception, the olfactometer is constructed of off-the-shelf parts used primarily in gas chromatography. The only portion of the device that requires adaptation is the odor cylinders. These Teflon cylinders are sold as one-way or check valves for chromatography applications (Cole-Parmer) and contain a spring-loaded silicone valve. By unscrewing the cylinder, the valve assembly is easily removed, leaving a $6 \times 10 \mathrm{~mm}$ cylindrical opening (see Figure 2). This is the area that will contain the odor-saturated filter paper. The ends of the Teflon cylinder are OmniFit fittings. A cross-section through this valve reveals that the interior of the fittings is an inverted cone. Because of this configuration, a 4-mm glass ball (Hoover Precision Products, Sault Ste. Marie, MI) placed inside the cone creates a ball valve. In operation, this ball valve prevents the odor from escaping into the nosepiece as long as the odor cylinder is upright in orientation and no positive pressure is present. Because, during high flow administration, the ball may be pushed so far upward that it occludes the exit tubing, a C-shaped ring is placed inside this fitting (see Figure 2). This $\mathrm{C}$ shaped piece is created by cutting a 1.5 -mm-long arc section from a ring used in the OmniFit fittings. This ensures that the ball is stopped before it closes the exit and leaves sufficient room for outward air flow.

To prevent the odor from diffusing down the long section of tubing (toward the flow controller), a second ball valve is needed upstream of the odor. This is accomplished by drilling a cone-shaped opening in the Teflon and inserting a 4-mm glass ball. Since the Teflon is rather soft and easily machined, a sharp spade-type drill bit ( $0.25 \mathrm{in}$.) can be used. Although this is a straightforward process, the conical opening must be symmetrical and smooth to ensure that the ball will perfectly occlude the opening.

The other portions of the olfactometer are easily assembled, using compression, flange, and hose barb fittings. Tygon tubing (7-mm inner diam) is used to carry air from the air pump to the filter and then to the flow valves. At this point in the system, an activated charcoalfilled canister may be used to wash the incoming air, in order to remove room air odors. The investigator should take care, however, to include several graded particulate filters downstream of the charcoal, to eliminate the associated charcoal dust.

The flowmeters chosen for this application were directreading $150-\mathrm{mm}$ glass float flowmeters $(2 \mathrm{~L} / \mathrm{min}$ maxi- mum flow) with built-in flow valves (Cole Parmer). These meters offer good precision and are factory calibrated. Mass flow controllers would increase the precision of the flow and could easily be substituted, if the investigator's budget permits.

At the output of the flow valves, Teflon tubing (1.6-mm inner diam) and fittings are used. The flow valves are connected to the three-way Teflon solenoid valves $(12 \mathrm{~V} \mathrm{dc}$; Cole Parmer), which provide the switched airstreams to the odor cylinders. All of the flowmeters and solenoids are housed inside a metal chassis $(54 \times 40 \times 35 \mathrm{~cm}$, width $\times$ height $\times$ depth). The long tubing (7 $\mathrm{m}$ in this model) leaving the metal chassis is placed inside a $32-\mathrm{mm}$ outer diameter hose. This hose leads to the four odor cylinders and the Teflon manifold, which are housed in a cylindrical plastic container ( $85 \mathrm{~mm}$ in diameter) placed near the subject's nose. Because of the mechanical ball valves, this cylinder must always be oriented in an upright position.

The output of the manifold may be transmitted to the subject in a number of ways, depending on the demands of the experiment. A single tube may be used as a nasal cannula, or a $\mathrm{Y}$ adapter may be used for dirhinic administration. The effluent may also be fitted with a lightweight oxygen mask.

The solenoid valves are controlled by a computercontrolled 12-VDC relay driver and relays (KeithleyMetrabyte, Staunton, MA, PIO-12 and ERA-8). During normal operation (no stimulation), air from the flowmeters associated with an odor channel enters two different solenoids and is vented into the room air. Air from the control flowmeter enters a single solenoid and is transmitted into the long tubing leading to the subject. During stimulation, all three solenoids are turned on. This causes the control flow to be vented into the room and the odor channels to be transmitted through the odor chambers and to the subject. Because the control flow equals the odor flow, no change in flow is present at the subject.

Software for this system will depend on the controller but is exceedingly simple for the PC-based KeithleyMetrabyte boards. In order to set the board for control of the relays, data is written to the control register memory locations that the board occupies in the computer. This initialization sequence can be implemented by sending two lines of BASIC code (other languages that allow direct memory addressing may also be used); the investigator need only send additional data to the memory location corresponding to the channels (port) to turn on and off various channels. We use a BASIC OUT statement to turn on the channel and then to turn it off after the administration interval. For instance, the following code fragment turns on odor channel 2 for $1 \mathrm{sec}$ :

$$
\begin{aligned}
& \text { OUT port, } 10 \\
& \text { SLEEP } 1 \\
& \text { OUT port, } 0
\end{aligned}
$$

where port is the address of the relay driver board control port. The argument of 10 for port in the first line turns 


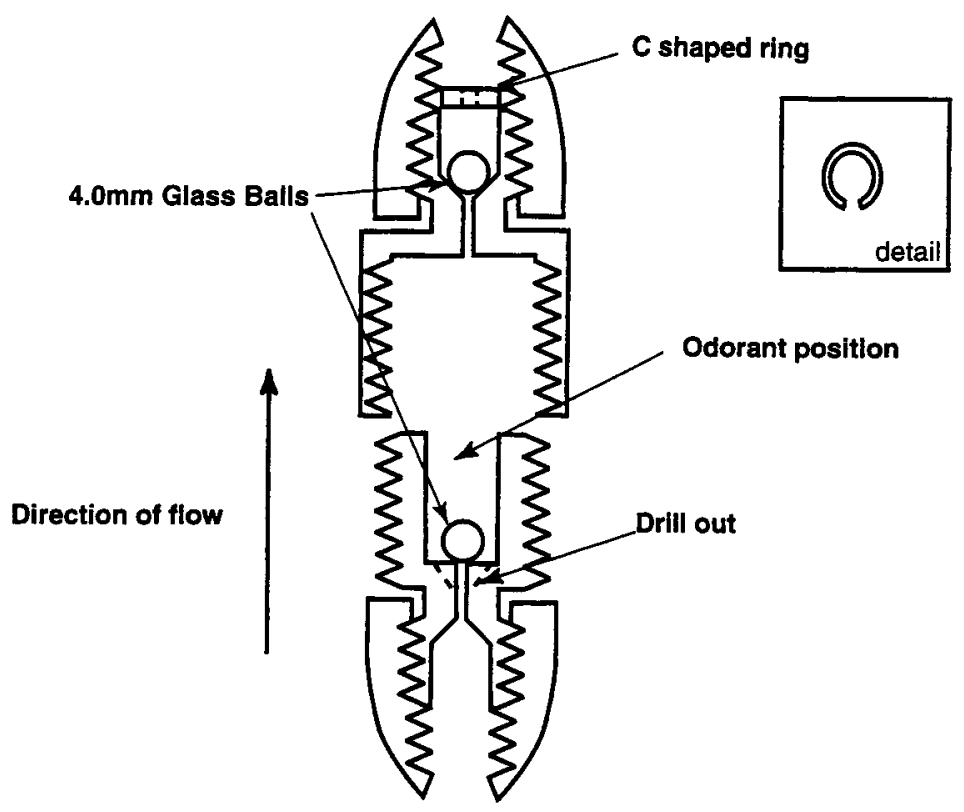

Figure 2. Detail of the odor cylinder/valve assembly.

on both odor channel 2 (2) and the control channel (8). Because the control channel is a normally open valve, its activation stops air flow through this valve. An argument of 9 would turn on channel 1 (1) and the control channel (8), and an argument of 12 would turn on channel 3 (4) and the control channel (8). The last line turns all the channels off. Other, more precise timing routines may, of course, be substituted for SLEEP. Given the rise time of the odorant in this device, the system clock provides adequate timing for most applications.

\section{NORMAL USE}

The number of channels possible with this type of olfactometer is limited only by practical concerns, such as the size of the device and the available air supply. In this application, an olfactometer capable of presenting three different odors was constructed. This resulted in a panel of eight flowmeters (two for each odor channel and one each for the control and constant channels). Seven threeway solenoid valves and four odor cylinders were used. Three odor cylinders were connected to the three odor channels, and the fourth cylinder contained the diluent (mineral oil) for the odors and was connected to the control solenoid channel.

Odorant materials for this olfactometer must be placed inside the odor cylinders. In our application, this was accomplished by placing $1 \mathrm{ml}$ of odors in solution on $1 \times$ $0.6 \mathrm{~cm}$ sections of perfumers' strips (precut blotter paper available from Aldrich). These strips were bent to form a V shape and placed in the cylinders.

In order to test the timing of the olfactometer, chilled $\left(0{ }^{\circ} \mathrm{C}\right)$ metal was placed in the odor cylinder, instead of filter paper. Total flow rate was $3 \mathrm{~L} / \mathrm{min}$, with $2.5 \mathrm{~L} / \mathrm{min}$ passing through the odor cylinder. A fast thermocouple (time constant $=0.00005$ ) was placed in the effluent at the nosepiece. This was interfaced to dc amplifiers (Neuroscan Synamps). Five stimulations lasting $1 \mathrm{sec}$ were presented and averaged. The response of the thermocouple is presented in Figure 3. In order to account for the response delay of the thermocouple, its response was recorded as it was placed in iced water. This indicated that thermocouple cooling took $172 \mathrm{msec}$ to reach $70 \%$ of maximum. Subtracting this time from the response to cold air stimulation indicated that the actual time for the cold air bolus to reach $70 \%$ of maximum (based on the suggestions of Evans, Kobal, Lorig, \& Prah, 1993) was 163 msec. Figure 3 illustrates both the raw thermocouple recording and a trace adjusted to account for the thermocouple's inertia.

Because the odorant airstream is unwarmed and presented at room humidity, high flow rates into the nose are not advisable. Subjects exposed to constant unhumidified flows higher than $3 \mathrm{~L} / \mathrm{min}$ often report nasal drying and discomfort. Even flows of $3 \mathrm{~L} / \mathrm{min}$ presented directly to the nares will produce discomfort and potential drying artifacts if maintained for more than $15 \mathrm{sec}$ at a time. For this reason, lower flow rates are suggested if the flow will be directed into the nostrils by means of a cannula. If a mask is used, flow rates slightly higher than $3 \mathrm{~L} / \mathrm{min}$ may be used without producing discomfort in the subject, although the configuration of the mask can introduce additional air flow or turbulence, affecting rise time. This factor should be a constant error, however.

One difficulty in the use of this olfactometer is the low volume of odorant solution that can be placed in the odor cylinder. If a large volume of air is passed through the cylinder, through either hundreds of short exposures or a few exposures of many seconds, the volume of odorant 


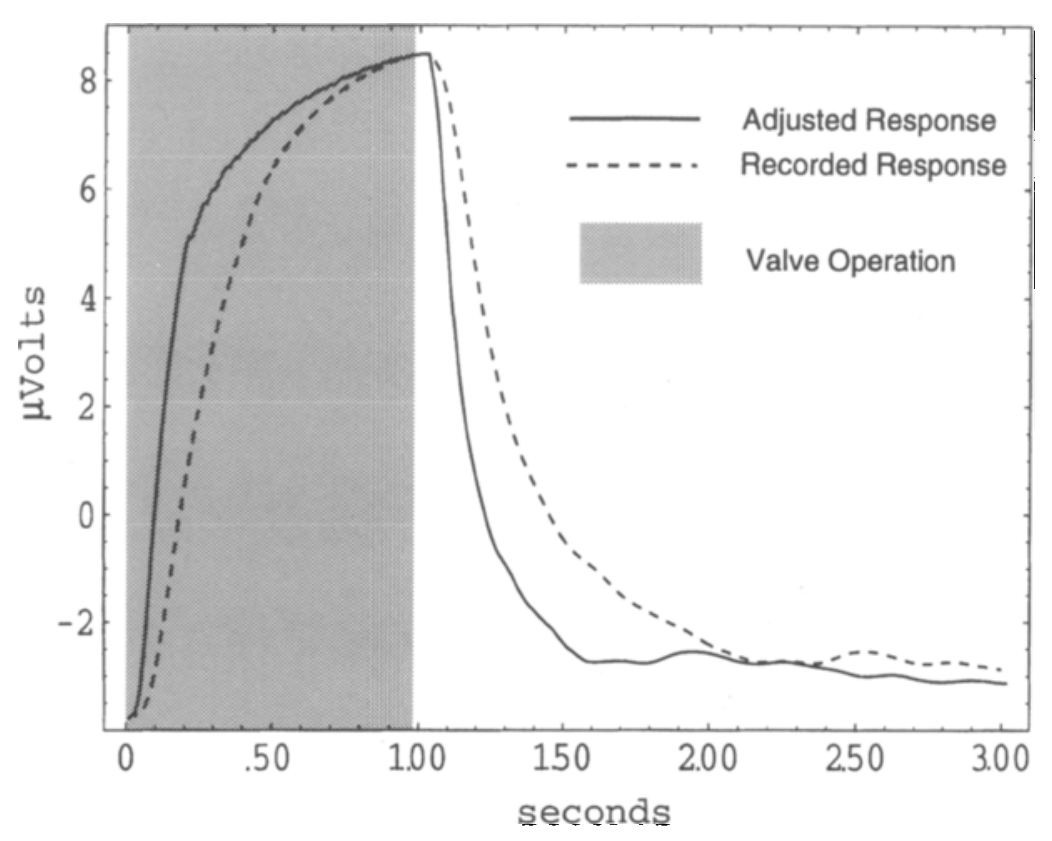

Figure 3. Thermocouple recording of the olfactometer output during 1-sec stimulation. Cool air, rather than odorant, was used in the odor cylinder. The solid line indicates the response of the olfactometer, adjusted to account for the thermocouple's inertia.

will be reduced, and this will subsequently reduce the concentration of odor in the bolus presented to the subject. This situation can be avoided if the odorant material is often refreshed in the odor cylinders. Refreshing the odorant can be accomplished by removing the cylinder from the long tubing and inserting a pipette into the Omnifit opening. By turning the cylinder upside down, a few milliliters of odorant can be pipetted past the glass ball and onto the paper. The frequency with which an investigator must refresh the odor cylinders will depend on the amount of air passed through the cylinder and the volatility of the particular odorant. Materials such as acetone would require frequent refreshing, whereas acetic acid would require fewer and less frequent reinoculations. Some odorants, because of oxidation, will change odor characteristics even though not depleted. In these cases, the odorant source must be replaced. Obviously, this olfactometer design is incapable of using gaseous odorants without substantial modification.

The only portions of the olfactometer requiring cleaning are the odor cylinders, the manifold, and the tubing connecting them. After removing the cylinders from the long tubing, they can be cleaned in an ultrasonic cleaner or by soaking. Since the long tubing segments have passed only clean air, they should not require cleaning. The same is true for the solenoids and flowmeters.

\section{OPERATIONAL EXAMPLE}

To demonstrate the type of data that can be obtained with this device, CSERPs were collected from a subject exposed to $4 \%, 2 \%$, and $0 \%$ butanol solutions (water diluent). The subject was a right-handed, 20-year-old male, who gave informed consent prior to data collection. Electrophysiological data $(0-1000 \mathrm{~Hz})$ were collected from 30 scalp locations, using an Electrocap with tin electrodes. The electroocculogram was also collected from the right eye, to correct for eye movements. Signal amplification was accomplished with a Neuroscan SynAmp. The subject was exposed to 16 trials at each concentration, with a mean interstimulus interval of $30 \mathrm{sec}$. Stimulus duration was $0.5 \mathrm{sec}$ and commenced after a 0.2 -sec baseline. The odorants were administered in randomized order and synchronized (via computer) with the onset of subject's inhalations. Breathing was monitored via a respiration belt (Grass Instruments). The total flow rate was $3 \mathrm{~L} / \mathrm{min}$. During stimulation, the odorant flow was $2 \mathrm{~L} / \mathrm{min}$, mixed with $1 \mathrm{~L} /$ min of clean air. The effluent of the olfactometer was positioned $2-3 \mathrm{~cm}$ under the subject's nose. This position was monitored by the experimenter via video camera. The subject was asked to count the total number of odors presented in each block of 16 trials, and a 2-min rest period was given between blocks.

After data collection, the individual trials were corrected for eye movements, baseline corrected, smoothed to $20 \mathrm{~Hz}$, and averaged for responses to each odorant and electrode. The averages for electrode $\mathrm{Cz}$ are presented in Figure 4. A clear CSERP is observable for both the $4 \%$ and the $2 \%$ butanol concentrations, whereas the $0 \%$ condition is relatively flat. The small negativity (peaking at $400 \mathrm{msec}$ poststimulus) followed by the large positivity (at $600 \mathrm{msec}$ poststimulus) is typical of breathing- 


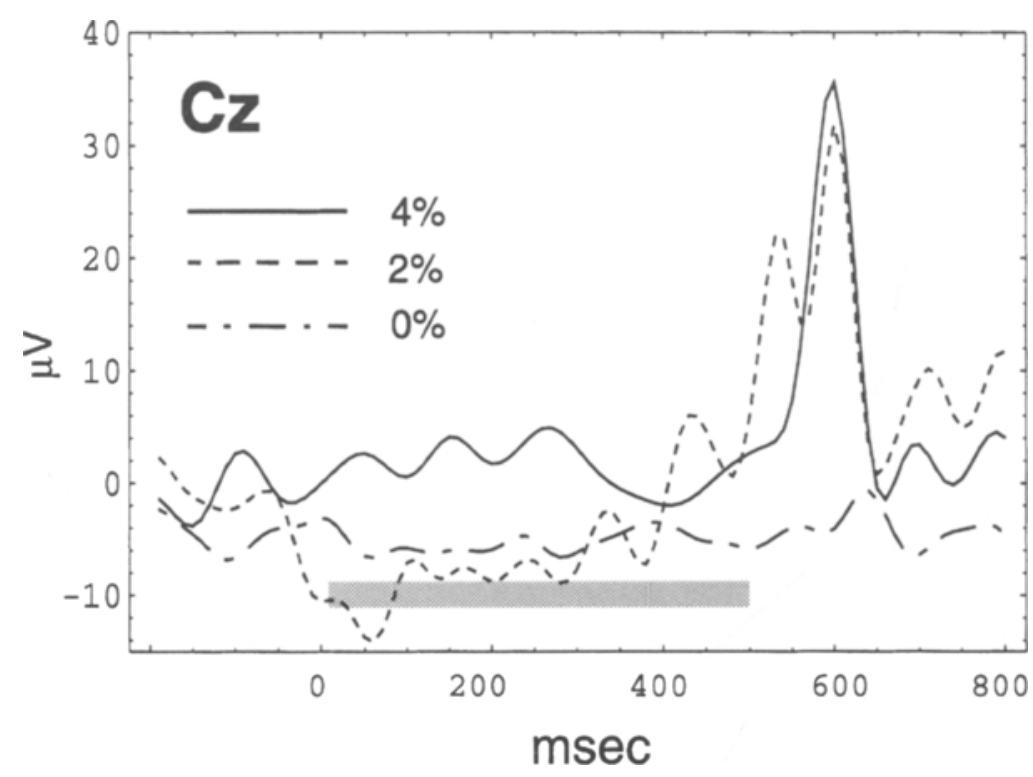

Figure 4. Chemosensory event-related potentials to different concentrations of butanol in a single subject recorded at $\mathrm{Cz}$. The bar indicates stimulus delivery timing.

synchronized (active) CSERPs (Lorig, Matia, Peszka, \& Bryant, 1996). This negativity tends to be larger when stimuli are presented with rapid rise times and are administered asynchronously with breathing.

At this time, parts for the olfactometer (excluding the air supply) cost about $\$ 3,500$. Designs omitting the air dilution channel should cost substantially less, in which case the experimenter must adjust the concentration of the odorant solution in order to adjust odor intensity. Other olfactometers used for CSERPs that offer multiple odorant channels, faster rise times, and humidification systems often cost in excess of $\$ 100,000$. Although extremely simple in comparison, the olfactometer described here has several advantages, including its nonferrous construction and resistance to contamination. In addition, the fast rise times offered by other olfactometers are unimportant for the current demands of fMRI use, since temporal resolution of this technique depends on the relatively slow nature of the hemodynamic response.

\section{REFERENCES}

Evans, W. J., Kobal, G., Lorig, T. S., \& Prah, J. (1993). Suggestions for collection and reporting of chemosensory (olfactory) eventrelated potentials. Chemical Senses, 18, 751-756.

Koвal, G., \& Hummel, T. (1988). Cerebral chemosensory evoked potentials elicited by chemical stimulation of the human olfactory and nasal mucosa. Electroencephalography \& Clinical Neurophysiology, 71, 241-250.

LAING, D. G. (1986). Identification of single dissimilar odors is achieved with a single sniff. Physiology \& Behavior, 37, 163-170.

Lorig, T. S., Matia, D. C., Peszka, J. J., \& Bryant, D. N. (1996). The effects of active and passive stimulation on chemosensory eventrelated potentials. International Journal of Psychophysiology, 23, 199-205.

Prah, J. D., Sears, S. B., \& Walker, J. C. (1995). Modern approaches to air dilution olfactometry. In R. Doty (Ed.), Handbook of olfaction and gustation (pp. 227-256). New York: Marcel Dekker.

(Manuscript received November 19, 1997; revision accepted for publication May 19,1998 .) 